\title{
Wearable technology in healthcare
}

Recent patents related to wearable devices and systems for monitoring glucose levels and other health conditions.

\begin{abstract}
Patent number Description
US 10,219,753 A wearable patch and method for automatically monitoring, screening, and/or reporting events related to one or more health conditions (e.g., sleeping, arrhythmias, breathing disorders, metabolic and nutritional status, glucose monitoring, lipid monitoring, type and intensity of physical activity, calorimetry) with on-board embedded processing for anomaly detection.

US 10,219,713 An apparatus and method to collect and analyze phonocardiogram (PCG) and electrocardiogram (ECG) waveforms. A wearable device attached to a patient collects heartbeat waveforms and sends the collected waveforms to a receiver through a wireless network. An acoustic seal layer is attached to the wearable device to reduce body movement noises and environmental noises. An analysis method compares the received patient's current PCG/ECG waveform with historic data, which can be stored in a cloud-based database.

US $10,188,325$

Wearable systems for noninvasive glucose sensing, which include an ultrasound generator, an ultrasound detector and a feedback unit. Methods for noninvasive glucose sensing using a wearable device include measuring a thickness (geometrical and/or optical) of a target tissue or a time of flight of ultrasound or optical pulses in the target tissue and determining a glucose value from the thickness of the target tissue or the time of flight in the target tissue in accordance with a target tissue thickness (geometric and/or optical) or time of flight versus glucose calibration curve.
\end{abstract}

US 10,188,299 A wearable device for measuring physiological parameters, including a light source having semiconductor light emitting diodes (LEDs) each configured to generate an output optical beam, wherein at least a portion of the one or more optical beam wavelengths is a near-infrared wavelength. The light source is configured to increase signal-to-noise ratio by increasing light intensity for at least one of the LEDs and by increasing a pulse rate of at least one of the LEDs. A lens is configured to receive the output optical beam and to deliver a lens output beam to tissue. A detection system generates an output signal in response to the lens output beam reflected from the tissue.

US 10,182,726 Continuous monitoring of a plurality of physiological data, which may be used for health and fitness improvements for a user. A physiological monitoring and measurement device may include a wearable strap that receives heart rate data for a user including a time series of heart rate data, a maximum heart rate, and a resting heart rate. A processor may transform the heart rate data into a time series of heart rate reserve data that is weighted, e.g., to account for cardiovascular efficiencies at different intensity levels, to provide a weighted time series of heart rate reserve values. An intensity score that provides an indicator of cardiovascular intensity for an exercise routine may be generated from the weighted time series of heart rate reserve values and displayed to the user on the wearable strap.

US 10,143,840 A wearable device for suppressing appetite or hunger in a patient, including a microprocessor, electrical stimulator and at least one electrode configured to deliver electrical stimulation to the epidermis, through a range of $0.1 \mathrm{~mm}$ to $10 \mathrm{~mm}$ or a range of $0.1 \mathrm{~mm}$ to $20 \mathrm{~mm}$ of the dermis, of a T2 frontal thoracic dermatome to a T12 frontal thoracic dermatome or meridian of the patient and/or front or back, C5-T1 dermatome across the hand and/or arm, and/or the upper chest regions. The device includes a pad, in which the electrode is disposed, for secure placement of the device on a skin surface of a patient. The device is adapted to provide electrical stimulation as per stimulation protocols and to communicate wirelessly with a companion control device configured to monitor and record appetite patterns of the patient. The control device is also configured to monitor, record, and modify parameters of the stimulation protocols.

US $10,136,85$

A wearable system and methods for measuring physiological data from a device worn about a body part of a user, comprising a base module, a first sensor module, and a second sensor module. The base module comprises a display and a base computing unit. The first sensor module measures a gravitational force experienced by the device. The second sensor module is spatially positioned relative to the base module and over a portion of the body part for measuring one or more physiological characteristics calibrated on the basis of the gravitational force measured with the first sensor module. The base module is adjustably positioned by the user relative to the second sensor module such that the sensor module maintains its positioning over the body part for sufficient contact with the body part for accurate measurements of physiological data regardless of the anthropometric size of the body part.

\begin{tabular}{lll} 
Assignee & Inventor & Date \\
\hline $\begin{array}{l}\text { Zansors } \\
\text { (McLean, }\end{array}$ & Dasgupta A, Das R & $3 / 5 / 2019$ \\
VA, USA) & & \\
$\begin{array}{l}\text { Bayland } \\
\text { Scientific } \\
\text { (Fremont, }\end{array}$ & Du X, Zhou Y & $3 / 5 / 2019$ \\
CA, USA) & & \\
& & \\
Esenaliev R & Esenaliev R & $1 / 29 / 2019$
\end{tabular}

Omni Medsci Islam MN

$1 / 29 / 2019$ (Ann Arbor, Ml, USA)

Whoop (Boston, Ahmed W,

$1 / 22 / 2019$ MA, USA) Capodilupo J, Nicolae A

Elira (St. Louis,

Perez RE, Goode

$12 / 4 / 2018$ MO, USA)

PV, Hong PI, Diianni S, Malave LJ, Stengel B, Faul JL

Source: United States Patent and Trademark Office (http://www.uspto.gov).

Published online: 2 April 2019

https://doi.org/10.1038/s41587-019-0093-3 